\title{
Ocular Changes in Diabetes Mellitus
}

\section{Partha Haradhan Chowdhury1*, Brinda Haren Shah² and Nripesh}

\section{Tiwari ${ }^{3}$}

1M.Optom, Associate Professor, Principal, Department of Optometry, Shree Satchandi Jankalyan Samiti Netra Prasikshan Sansthan, Pauri, Affiliated to Uttarakhand State Medical Faculty, Dehradun, India

2M.Optom, Guest Lecturer, Department of Optometry, Shree Satchandi Jankalyan Samiti Netra Prasikshan Sansthan, Pauri, Affiliated to Uttarakhand State Medical Faculty, Dehradun, India

${ }^{3}$ D.Optom, General Secretary and Chief Optometrist, Department of Optometry, Shree Satchandi Jankalyan Samiti Netra Prasikshan Sansthan, Pauri, Affiliated to Uttarakhand State Medical Faculty, Dehradun, India

*Corresponding author: Partha Haradhan Chowdhury, M. Optom, Associate Professor, Principal, Department of Optometry, Shree Satchandi Jankalyan Samiti Netra Prasikshan Sansthan, Pauri, Affiliated to Uttarakhand State Medical Faculty, Dehradun, India, Email: optometrypublish@gmail.com

\section{Abstract}

This paper describes about diabetes mellitus and its effect on different parts of the eye.

\section{Introduction}

Diabetes Mellitus crucially affects the eye. Most of the time it is considered as a silent killer. It's another name is Micro Angiopathy. Micro = small, Angio = blood vessels and Pathy $=$ disease.

In case of Diabetes Mellitus, all parts of the eye are affected. In eyelid, there are chances of trichiasis, ptosis etc. In conjunctiva, hyperaemia, conjunctivitis, itchiness, mild burning sensation etc. In Sclera, mild congestion and redness. In Iris, chances of Iris Atrophy and Tremolousness of Iris. In Vitreous, vitreous detachment and in pupil, sluugishness of pupil during exposure of light.

Retina is affected most in cases of Diabetes Mellitus. About 4-28\% of the population is affected by Diabetic Retinopathy and $2 \%$ becomes blind due to this cause.
For Diabetic Retinopathy, two considerable factors are: severity and duration

Types:

- IDDM - Insulin Dependent Diabetes Mellitus

- NIDDM - Non Insulin Dependent Diabetes Mellitus

\section{Pathogenesis}

It's another name is Micro Angiopathy. This micro angiopathy creates occlusion and this occlusion can create leakage and ischemia. Further hypoxia is developed and it will lead to Neovascularization and AV shunt. Usually, leakage mainly occurs due to weakness of the wall of the blood vessels. It mainly occurs due to loss of pericytes. Ultimately, it develops edema and haemorrhage [1-3].

\section{Stages}

A. Non Proliferative Diabetic Retinopathy

B. Clinically significant Macular Edema

C. Proliferative Diabetic Retinopathy

D. Advanced Proliferative Diabetic Retinopathy 


\section{Open Access Journal of Ophthalmology}

\section{A. Non Proliferative Diabetic Retinopathy}

It is the first stage of Diabetic Retinopathy. It is considered when signs like microaneurysms, small dot and blot haemorrhage, IRMA and cotton wool spots are present.

\section{B. Clinically significant Macular Edema}

It is considered when Retinal Thickness is greater than $500 \mu \mathrm{m}$. It is diagnosed with OCT.

\section{Proliferative Diabetic Retinopathy}

It is considered when Neovascularization is developed in Retina. At first, Neo vascularization elsewhere (NVE) is developed. When NVE is developed in $1 / 4^{\text {th }}$ part of the Retina, NVD (Neo vascularization on Disc) is created. 5\% of Diabetic population is involved in Proliferative Diabetic Retinopathy. In this case, FFA is very essential to find out the leakage. With increasing PDR, there are chances of Vitreous Detachment and Haemorrhages.

\section{Advanced Proliferative Diabetic Retinopathy}

It is considered when, consequently vitreous haemorrhage, Tractional Retinal Detachment and burn out Proliferative Diabetic Retinopathy occurs.

\section{References}

1. Brad Bowling (2016) Kanski's Clinical Ophthalmology E-Book: A Systematic Approach. $8^{\text {th }}$ (Edn.).

2. Sihota, Radhika Tandon (2018) Parson's Diseases of the Eye. $22^{\text {nd }}$ (End.).

3. Samar K Basak (2009) Clinical Ophthalmology.

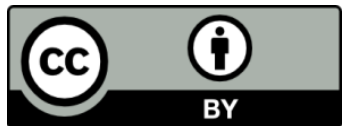

